\title{
IGR J16393-4643: a new heavily-obscured X-ray pulsar
}

\author{
A. Bodaghee ${ }^{1,2}$, R. Walter ${ }^{1,2}$, J. A. Zurita Heras ${ }^{1,2}$, A. J. Bird ${ }^{3}$, T. J.-L. Courvoisier ${ }^{1,2}$, A. Malizia ${ }^{4}$, \\ R. Terrier ${ }^{5,6}$, and P. Ubertini ${ }^{7}$
}

\author{
1 INTEGRAL Science Data Centre, Chemin d'Ecogia 16, 1290 Versoix, Switzerland \\ e-mail: arash . bodaghee@obs . unige.ch \\ 2 Observatoire de Genève, Chemin des Maillettes 51, 1290 Sauverny, Switzerland \\ 3 School of Physics and Astronomy, University of Southampton, Highfield, Southampton, SO17 1BJ, UK \\ 4 IASF/CNR, Via Gobetti 101, 40129 Bologna, Italy \\ 5 CEA-Saclay, DAPNIA/Service d'Astrophysique, 91191 Gif-sur-Yvette Cedex, France \\ ${ }^{6}$ Fédération de recherche APC, Collège de France, 11 place Marcelin Berthelot, 75231 Paris, France \\ 7 IASF/CNR, Via Fosso del Cavaliere 100, 00133 Rome, Italy
}

Received 11 July 2005 / Accepted 27 September 2005

\section{ABSTRACT}

An analysis of the high-energy emission from IGR J16393-4643 (=AXJ1639.0-4642) is presented using data from INTEGRAL and $X M M$-Newton. The source is persistent in the $20-40 \mathrm{keV}$ band at an average flux of $5.1 \times 10^{-11} \mathrm{erg} \mathrm{cm}^{-2} \mathrm{~s}^{-1}$, with variations in intensity by at least an order of magnitude. A pulse period of $912.0 \pm 0.1 \mathrm{~s}$ was discovered in the ISGRI and EPIC light curves. The source spectrum is a strongly-absorbed $\left(N_{\mathrm{H}}=(2.5 \pm 0.2) \times 10^{23} \mathrm{~cm}^{-2}\right)$ power law that features a high-energy cutoff above $10 \mathrm{keV}$. Two iron emission lines at 6.4 and $7.1 \mathrm{keV}$, an iron absorption edge $\gtrsim 7.1 \mathrm{keV}$, and a soft excess emission of $7 \times 10^{-15} \mathrm{erg} \mathrm{cm}^{-2} \mathrm{~s}^{-1}$ between $0.5-2 \mathrm{keV}$, are detected in the EPIC spectrum. The shape of the spectrum does not change with the pulse. Its persistence, pulsation, and spectrum place IGR J16393-4643 among the class of heavily-absorbed HMXBs. The improved position from EPIC is RA $(\mathrm{J} 2000)=16^{\mathrm{h}} 39^{\mathrm{m}} 05.4^{\mathrm{s}}$ and $\mathrm{Dec}=-46^{\circ} 42^{\prime} 12^{\prime \prime}$ (4" uncertainty) which is compatible with that of 2MASS J16390535-4642137.

Key words. gamma-rays: observations - X-rays: binaries - pulsars: individuals: IGR J16393-4643 - pulsars: individuals: AX J1639.0-4642

\section{Introduction}

The INTEGRAL core program (CP: Winkler et al. 2003) routinely devotes observation time to Galactic Plane Scans (GPS) and Galactic Centre Deep Exposures (GCDE). These numerous snapshots of the Milky Way can be assembled into mosaic images of long exposure time ( $\sim 1 \mathrm{Ms})$. This gamma-ray view of the galaxy, as collected by ISGRI (Ubertini et al. 2003; Lebrun et al. 2003), enabled Bird et al. (2004) to detect 123 sources at a significance above $6 \sigma$. Around 20 of these sources are of unknown origin. A good portion of these new sources probably belong to the class of heavily-absorbed High Mass X-ray Binaries (HMXBs) that are concentrated along the galactic plane and in the spiral arms.

High-Mass X-ray Binaries are composed of a compact object such as a neutron star or a black hole that orbits a massive stellar companion. Depending on the type of companion, known HMXBs can be divided into two groups (van Paradijs 1983). Most HMXBs classified by Liu et al. (2000) contain a Be star. These systems are usually transient sources with hard spectra. The compact object has a wide orbit which mostly keeps it away from the Be star and its disk. Outbursts in these systems are due primarily to the compact object approaching the star and accreting matter from the slow, dense stellar wind. The second group of HMXBs features an O or B supergiant star. The orbit of the compact object places it well within the stellar wind, so material from the supergiant can be fed directly to the compact object through Bondi accretion, or it can pass to the compact object via an accretion disk. The latter mechanism is typically found in bright X-ray binaries in which the Roche lobe overflow of gas from the OB star supersedes the flow of accreting matter. For less luminous binaries, the OB star does not fill its Roche lobe and the behaviour of the X-ray source is determined predominantly by the stellar wind. X-ray emission in supergiant HMXB systems is usually persistent, with flares stemming from inhomogeneities in the wind. Neutron stars with strong magnetic fields develop a hot spot for accretion which can result in a pulsation.

The number of persistent and heavily-absorbed HMXBs associated with supergiant companions has increased thanks to deep, wide-field observations by INTEGRAL combined with follow-up X-ray monitoring by XMM-Newton (Walter et al. 2003; Rodriguez et al. 2003; Hill et al. 2005; Lutovinov et al. 2005b). So far, these sources have been detected preferentially in the Norma Arm region (Walter et al. 2004) which features high formation rates of OB supergiant stars. 


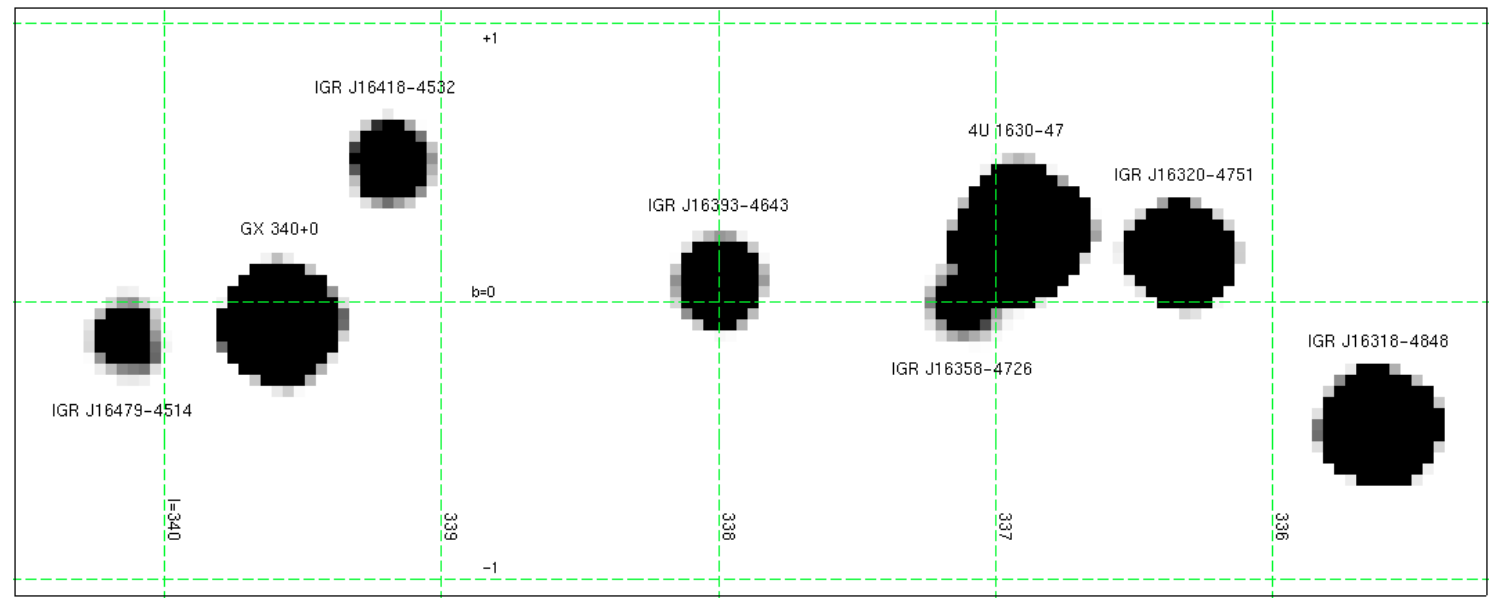

Fig. 1. Intensity map of the Norma Arm in the $20-40 \mathrm{keV}$ band accumulating data from revolutions $30-185$ for an effective exposure time of $670 \mathrm{ks}$. The image is in galactic coordinates and represents an area $2^{\circ}$ tall by $5^{\circ}$ wide. The galactic equator $(b=0)$ bisects the image horizontally. Neighboring sources from the catalog of Bird et al. (2004) are also shown.

It is there that INTEGRAL uncovered its first new source, IGR J16318-4848 (Walter et al. 2003). The Norma Arm also harbors IGR J16393-4643. This object was initially discovered in the X-ray band and listed as AX J163904-4642 in the ASCA Faint Source Catalog (Sugizaki et al. 2001). Its flat powerlaw slope, its absorption $\left(N_{\mathrm{H}}=13_{-7}^{+9} \times 10^{22} \mathrm{~cm}^{-2}\right)$, its lack of radio emission, and its position in the galactic plane compelled the authors to classify it as a HMXB. A non-thermal radio counterpart was recently detected in the ASCA error box which suggests a dust-enshrouded microquasar interpretation for the HMXB (Combi et al. 2004), and would help explain the possible association with the unidentified EGRET source 3EG J1639-4702 (Hartman et al. 1999) noted by Malizia et al. (2004).

We detected IGR J16393-4643 in the ISGRI GPS data of the Norma Arm's first visibility period, and we obtained a follow-up observation with XMM-Newton. The set of INTEGRAL and XMM-Newton data are introduced in Sect. 2. In Sect. 3, the refined X-ray position is used to locate the most likely counterpart at other wavelengths. Timing and spectral analyses are presented in Sects. 4 and 5, respectively. Finally, we discuss the nature of the source and we offer our conclusions in Sect. 6.

\section{Observations and data sets}

\subsection{INTEGRAL data and imaging}

The INTEGRAL data consist of all CP pointings during revolutions 30-260, as well as pointings that were public by January 3, 2005, which had the source within the ISGRI field of view (FOV). To improve the quality of the output mosaic, pointings with exposure times below $1 \mathrm{ks}$ were ignored. The resulting data set groups roughly 1500 pointings with an average exposure time of $2 \mathrm{ks}$ each.

Version 4.2 of the INTEGRAL Offline Scientific Analysis (OSA) software was used to reduce raw data into images. Source extraction employed version 18 of the INTEGRAL General Reference Catalog (Ebisawa et al. 2003) selected for objects detected by ISGRI. These tools are available to the public through the INTEGRAL Science Data Centre (ISDC: Courvoisier et al. 2003).

Intensity, significance, variance, and exposure mosaic images were constructed from background-subtracted images of individual pointings. Figure 1 provides an example of an intensity map of IGR J16393-4643 and its vicinity in the $20-40 \mathrm{keV}$ band from the pointings of revolutions 30 to 185 . The effective exposure time is $670 \mathrm{ks}$ after correcting for vignetting. Using this image, we extracted a source location of RA (J2000) = $16^{\mathrm{h}} 39^{\mathrm{m}} 05^{\mathrm{s}}$ and Dec $=-46^{\circ} 42.3^{\prime}\left(26^{\prime \prime}\right.$ uncertainty $)$ which agrees with and improves the ISGRI position of Bird et al. (2004). The mean flux $(20-60 \mathrm{keV})$ of the source is $0.73 \pm$ 0.02 counts per second (cps), or $4.9 \mathrm{mCrab}$, at a significance of $36 \sigma$.

\subsection{XMM-Newton data and imaging}

XMM-Newton (Jansen et al. 2001; Strüder et al. 2001; Turner et al. 2001) observed IGR J16393-4643 on March 21, 2004, from 08:21:15 to 11:41:15 (UT). We used the Science Analysis System (SAS) v. 6.1.0 software to analyse the data and to extract the EPIC spectrum. The data were screened for background variability by excluding time intervals in which the count rate above $10 \mathrm{keV}$ was greater than a selected threshold (0.33 cps for EPIC/MOS1, $0.25 \mathrm{cps}$ for EPIC/MOS2, and $2.4 \mathrm{cps}$ for $E P I C / P N)$. After screening, the effective exposure times were around $8.4,8$, and $7 \mathrm{ks}$ for EPIC/MOS1, $E P I C / M O S 2$, and $E P I C / P N$, respectively.

IGR J16393-4643 is clearly detected in images taken by EPIC. Figure 2 presents an image of IGR J16393-4643 from the EPIC/MOS1 camera. Furthermore, the source coincides with the updated ISGRI position and error circle from this paper. The refined X-ray position averaged from EPIC/MOS and $E P I C / P N$ is RA $(\mathrm{J} 2000)=16^{\mathrm{h}} 39^{\mathrm{m}} 05.4^{\mathrm{s}}$ and Dec $=$ $-46^{\circ} 42^{\prime} 12^{\prime \prime}$ ( $4^{\prime \prime}$ uncertainty). 


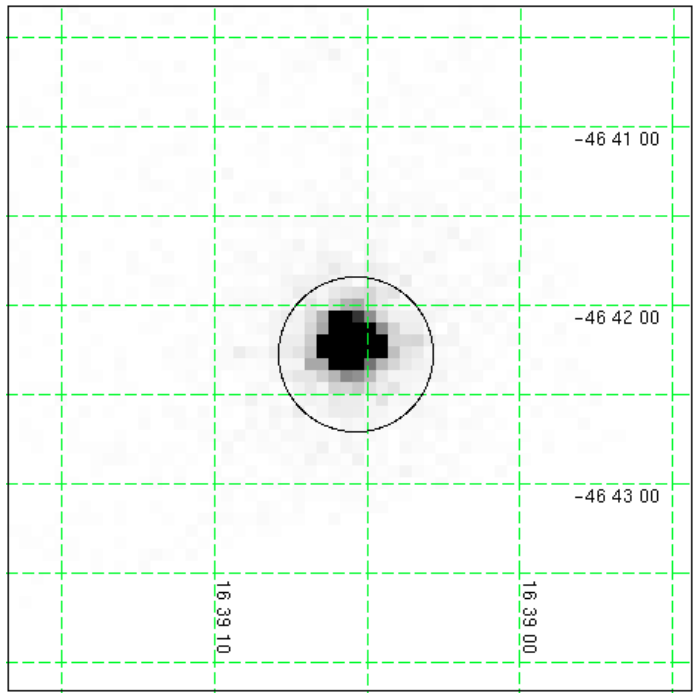

Fig. 2. EPIC/MOS1 image of IGR J16393-4643 in the $0.15-12 \mathrm{keV}$ energy range with an effective exposure time of $8.4 \mathrm{ks}$. The refined ISGRI error circle from this paper is superimposed.

\section{Counterparts}

We searched for counterparts at other wavelengths and found a single infrared source belonging to the Two Microns All-Sky Survey (2MASS: Cutri et al. 2003) in the XMM-Newton error box. This potential counterpart, 2MASS J16390535-4642137, is located about $2^{\prime \prime}$ away from the XMM-Newton position at RA $(\mathrm{J} 2000)=16^{\mathrm{h}} 39^{\mathrm{m}} 05.36^{\mathrm{s}}$ and Dec $=-46^{\circ} 42^{\prime} 13.7^{\prime \prime}$ $\left(0.06^{\prime \prime}\right.$ uncertainty). It appears in the $J, H$, and $K_{\mathrm{s}}$ bands with magnitudes of $14.63 \pm 0.06,13.32 \pm 0.04$, and $12.78 \pm 0.04$, respectively ( $95 \%$ confidence).

Figure 3 shows that the XMM-Newton position and error radius for IGR J16393-4643 do not intersect the error boxes of the radio source MOST J1639.0-4642 and the far infrared source IRAS 16353-4636, which are the possible counterparts proposed by Combi et al. (2004). The tight XMM-Newton error circle excludes optical counterparts from the USNO-B1 cata$\log$ (Monet et al. 2003), radio sources from the Vizier database (Ochsenbein et al. 2000), and ROSAT sources (Voges et al. 1999).

A possible association of IGR J16393-4643 with the EGRET source 3EGJ1639-4702 (Hartman et al. 1999) has been proposed by Malizia et al. (2004). Another ISGRI source, IGR J16358-4726, and 4U 1630-47 are just outside the EGRET $95 \%$ error contour. In the $2^{\circ} \times 5^{\circ}$ degree section of the Norma Arm presented in Fig. 1, the probability to observe a counterpart in the $0.56^{\circ}$ error radius of the EGRET source is close to 1 , so the coincidence with IGR J16393-4643 is probably a chance one.

\section{Timing analysis}

\subsection{Long-term variability}

Most ISGRI pointings have exposure times that are too brief for a significant detection. Therefore, a mosaic was prepared for each 3-day spacecraft revolution (from 30-244) in the 20-60

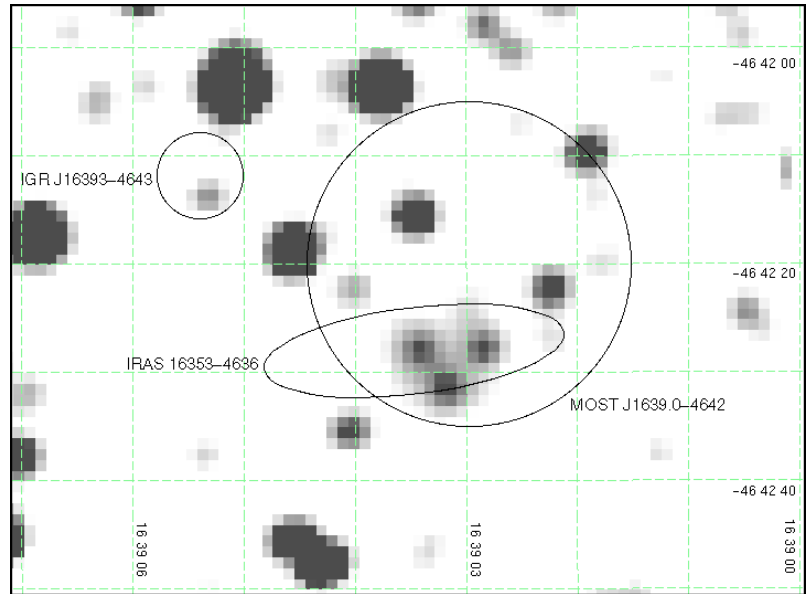

Fig. 3. Image from the 2MASS survey of the region around IGR J16393-4643 in the $K_{\mathrm{s}}$ band. The XMM-Newton error circle from this paper does not intersect the error boxes of the radio source MOST J1639.0-4642 and the far infrared source IRAS 16353-4636. The only infrared object inside the XMM-Newton error circle is 2MASS J16390535-4642137.

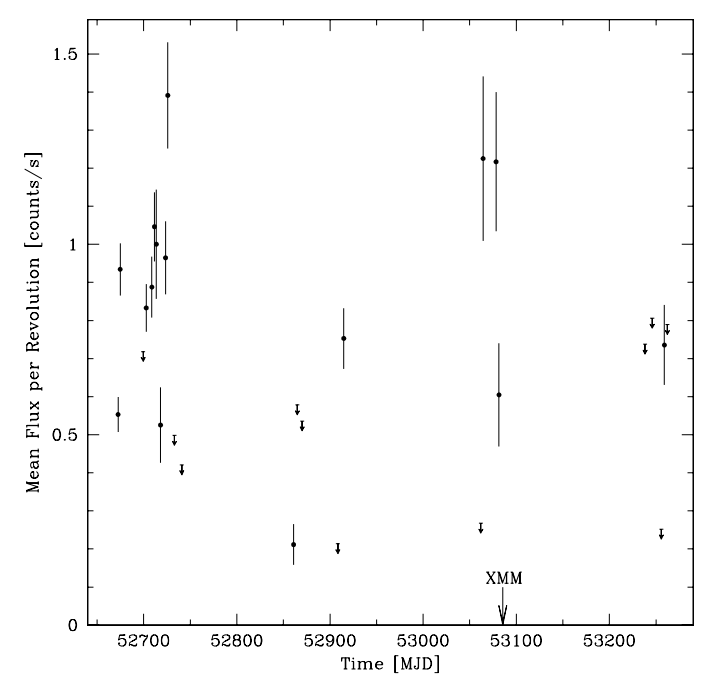

Fig. 4. ISGRI light curve (20-60 keV) of IGR J16393-4643 where each point represents the flux averaged over 1 spacecraft revolution. Upper limits $(3 \sigma)$ are provided when the source is not detected for an effective exposure time above $7 \mathrm{ks}$. The XMM-Newton observation (Fig. 5) occurred on MJD 53085.

and $60-150 \mathrm{keV}$ bands. We extracted the source flux, error, and significance by fitting a Gaussian with a fixed point spread function to the mosaic images. Upper limits $(3 \sigma)$, calculated from the variance maps, are provided when the source is not detected at the $4 \sigma$ level in a mosaic image with an effective exposure time above $7 \mathrm{ks}$. There were no detections in mosaic images of the 60-150 keV band so its light curve is omitted.

Figure 4 illustrates the persistence of IGR J16393-4643 in the $20-60 \mathrm{keV}$ energy range. Table 1 collects the 15 revolutions in which the source is detected in the mosaic image with a significance above $4 \sigma$. The average flux in these mosaics is $0.86 \mathrm{cps}(5.6 \mathrm{mCrab})$ with a cumulative exposure time of $1.5 \mathrm{Ms}$. The mean flux per revolution varies by a factor of at 


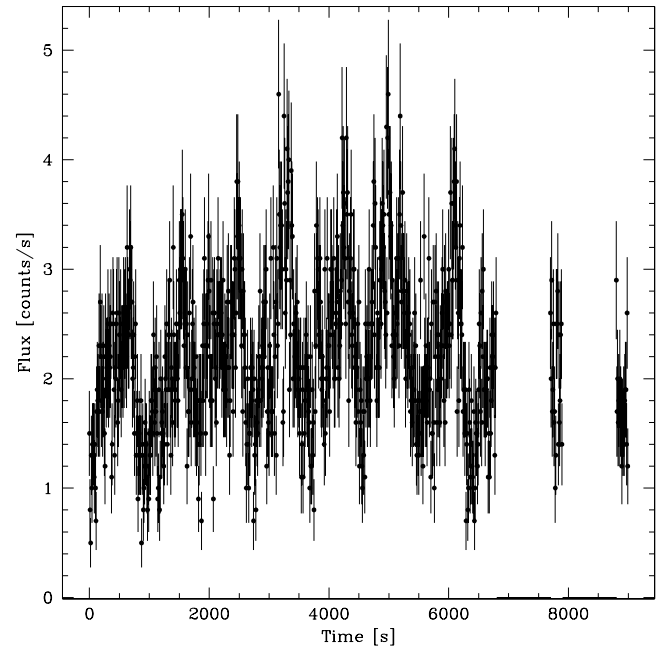

Fig. 5. EPIC/PN light curve for IGR J16393-4643 in the $2-10 \mathrm{keV}$ energy range with a time resolution of $10 \mathrm{~s}$. The observation starts at MJD 53 085.37516.

Table 1. Source flux value (20-60 keV), significance, average off-axis angle, and exposure time when IGR J16393-4643 is detected at the $4 \sigma$ level in the mosaic image of a spacecraft revolution. The median MJD of the visibility period is also given in reference to Fig. 4.

\begin{tabular}{|c|c|c|c|c|c|}
\hline \multicolumn{2}{|c|}{ Visibility period } & $\begin{array}{l}\text { Exp. } \\
{[\mathrm{ks}]}\end{array}$ & $\begin{array}{c}\text { Flux } \\
\text { [counts/s] }\end{array}$ & $\begin{array}{l}\text { Sig. } \\
{[\sigma]}\end{array}$ & $\begin{array}{c}\text { Angle } \\
{\left[{ }^{\circ}\right]}\end{array}$ \\
\hline 37 & 52672 & 204 & $0.55 \pm 0.05$ & 12.1 & 3.8 \\
\hline 38 & 52675 & 92 & $0.93 \pm 0.07$ & 13.7 & 3.6 \\
\hline 47 & 52703 & 153 & $0.83 \pm 0.06$ & 13.3 & 7.0 \\
\hline 49 & 52709 & 85 & $0.89 \pm 0.08$ & 11.1 & 6.2 \\
\hline 50 & 52712 & 104 & $1.05 \pm 0.09$ & 11.6 & 9.5 \\
\hline 51 & 52714 & 76 & $1.00 \pm 0.14$ & 7.0 & 11.3 \\
\hline 52 & 52718 & 93 & $0.53 \pm 0.10$ & 5.3 & 10.1 \\
\hline 54 & 52724 & 100 & $0.96 \pm 0.10$ & 10.0 & 9.7 \\
\hline 55 & 52726 & 96 & $1.39 \pm 0.14$ & 10.0 & 11.9 \\
\hline 100 & 52861 & 205 & $0.21 \pm 0.05$ & 4.0 & 6.9 \\
\hline 118 & 52915 & 146 & $0.75 \pm 0.08$ & 9.4 & 10.0 \\
\hline 168 & 53064 & 28 & $1.22 \pm 0.22$ & 5.7 & 11.5 \\
\hline 173 & 53078 & 27 & $1.22 \pm 0.18$ & 6.7 & 9.3 \\
\hline 174 & 53081 & 53 & $0.60 \pm 0.14$ & 4.5 & 9.3 \\
\hline 233 & 53259 & 72 & $0.74 \pm 0.10$ & 7.0 & 9.2 \\
\hline
\end{tabular}

least 6 from $0.21 \pm 0.05$ counts per second (cps) or $1.4 \mathrm{mCrab}$ in revolution $100(205 \mathrm{ks})$, to $1.39 \pm 0.14 \mathrm{cps}(9.3 \mathrm{mCrab})$ in revolution $55(96 \mathrm{ks})$. When the flux value during revolution 100 is compared to the highest flux registered in a single 2-ks pointing of the $20-60 \mathrm{keV}$ band $(4.3 \pm 0.6 \mathrm{cps}$, or $29 \mathrm{mCrab}$, during MJD 52673.623-52673.641), we find that the source flux varies by a factor larger than 20 .

\subsection{Pulsations}

During the $E P I C / P N$ observation of $8 \mathrm{ks}$, the source count rate varied from 1 to $4 \mathrm{cps}$ (Fig. 5). Furthermore, the variations occur periodically. By searching for modulations in the power spectrum and in the $\chi^{2}$ distribution, we obtain a period
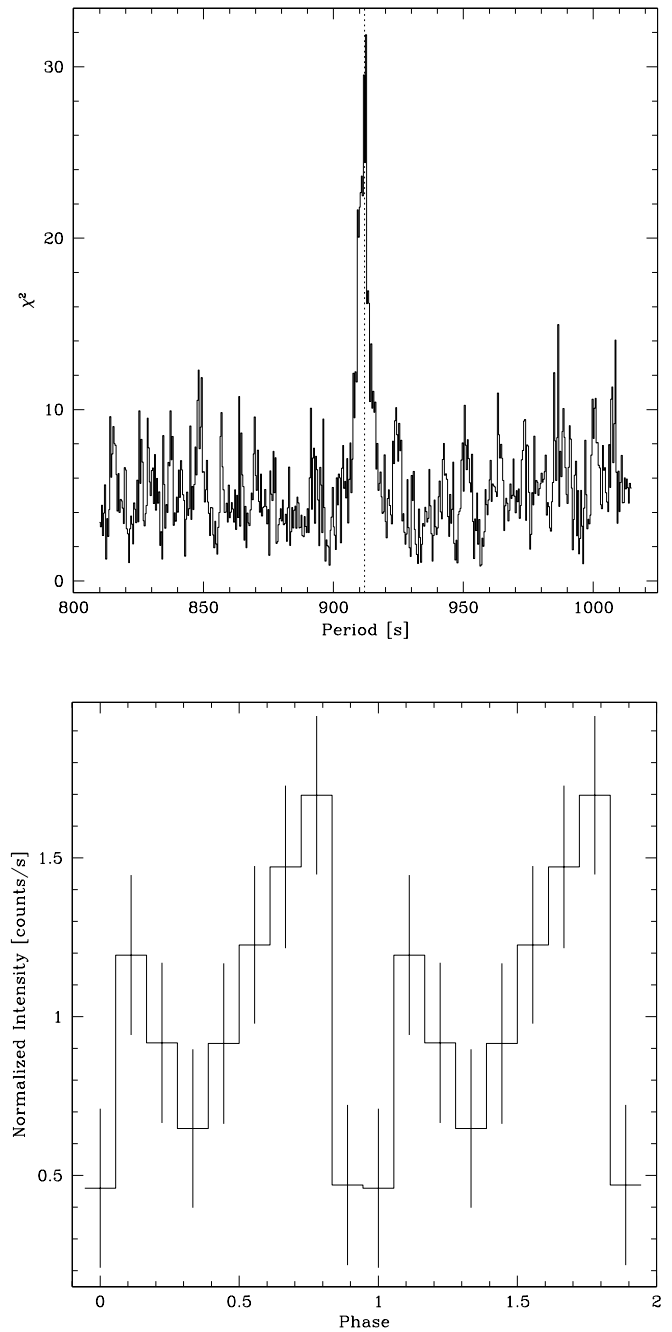

Fig. 6. Top a): period search ( $\chi^{2}$ distribution) on the ISGRI light curve (15-40 keV) of revolution 38, centred at $912 \mathrm{~s}$ (vertical line), with 9 bins per period, and a resolution of $0.4 \mathrm{~s}$. Bottom $\mathbf{b}$ ): pulse profile of the folded ISGRI light curve (15-40 keV) for a period of $912 \mathrm{~s}$. The start time is MJD 52674.53833.

of $912 \pm 5$ s at a $\chi^{2}$ of $\sim 600$ for 30 bins per period (Fig. 7a). The pulse period is determined by the centre of a Gaussian fit to the $\chi^{2}$ distribution, and the error is calculated using the procedure developed by Horne \& Baliunas (1986) on LombScargle periodograms. The EPIC/PN pulse profile folded with a period of $912 \mathrm{~s}$, illustrated in Fig. 7b, indicates a pulse fraction of $38 \pm 5 \%$.

For ISGRI, the pulse is best detected in the 100-s light curve of revolution 38 in the $15-40 \mathrm{keV}$ band since this revolution has enough effective exposure time $(50 \mathrm{ks})$ with low off-axis angles $\left(\sim 4^{\circ}\right)$, and its light curve is free of gaps that hinder a periodicity search. Figure 6 a presents the $\chi^{2}$ distribution centered at $912 \mathrm{~s}$, with 9 bins per period. The phase diagram is shown in Fig. 6b, and the pulse fraction is $57 \pm 24 \%$. We merged the 100-s light curves (15-30 keV) from revolutions 37-55, and derived a pulse period and error of $912.0 \pm 0.1 \mathrm{~s}$.

With respect to the pulse fractions, the amplitude does not change significantly as a function of energy. Both display 

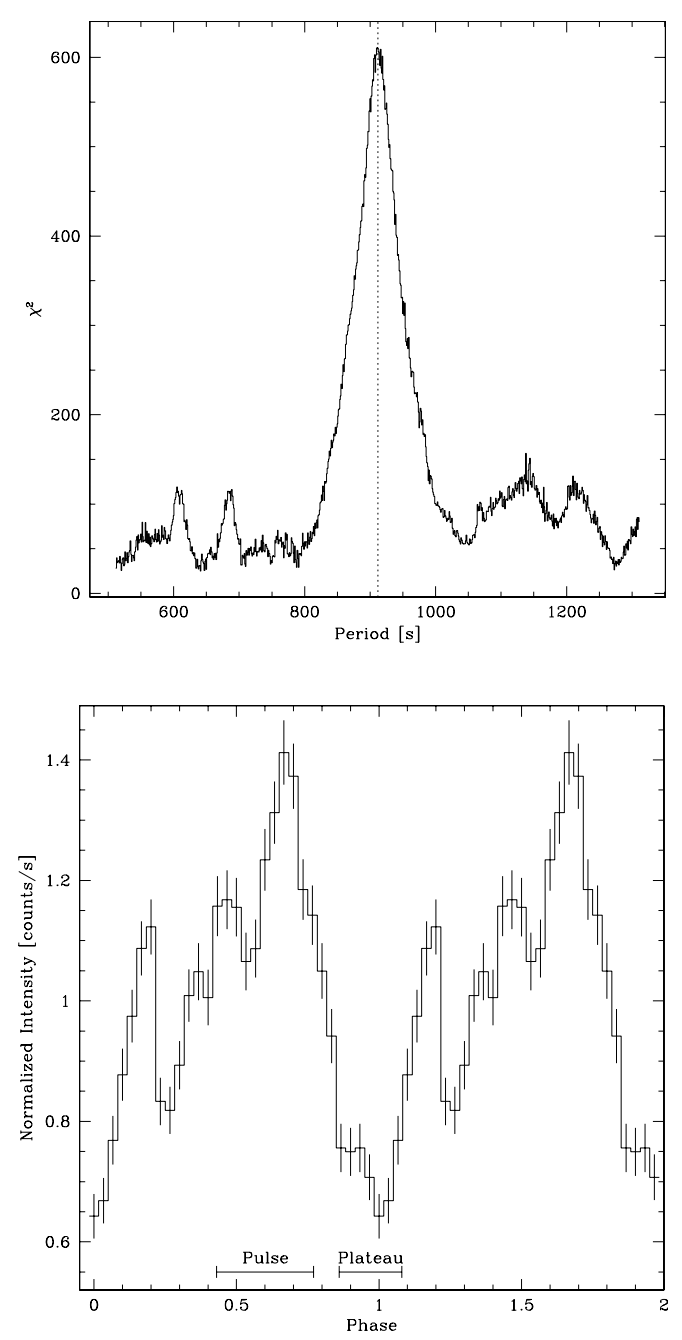

Fig. 7. Top a): distribution of $\chi^{2}$ for the EPIC/PN 2-10 keV light curve with 30 bins per period, a resolution of $1 \mathrm{~s}$, and centred at $912 \mathrm{~s}$ (vertical line). Bottom b): folded $E P I C / P N$ light curve for a pulse period of $912 \mathrm{~s}$ beginning at MJD 53085.38738 . The intervals indicate the pulse and plateau states of the phase-resolved spectrum.

a jagged rise to a peak flux followed by a drop. There appear to be primary and secondary peaks in the pulse profiles of both instruments at phases of $\sim 0.7$ and $\sim 0.2$, respectively. Although the ISGRI and EPIC periods are derived from observations about 400 days apart, the period is not accurate enough to search for possible variations. In addition, the ISGRI light curves of revolutions that are nearly concurrent with the EPIC observation present low source significances which makes it difficult to search for a period.

There is an indication in the EPIC light curve (Fig. 5) that the source varies over timescales longer than the pulse period. At the beginning of the observation, the maximum of the pulse is about 3 cps. This maximum rises to 4 cps about $5 \mathrm{ks}$ after the start time, and then decreases.

We did not detect an orbital period of the order of a few days in the combined ISGRI light curves (15-30 keV, 100-s resolution) of detected revolutions between 37 and 55 .

\section{Spectral analysis}

\subsection{Average spectrum}

To extract an ISGRI spectrum, we created mosaics accumulating the data from revolutions $37-185$ in the following energy bands; 22.09-25.92, 25.92-30.23, 30.23-40.28, 40.2851.29 , and 51.29-63.26 keV. These boundaries were chosen to conform with the current response matrices. The source is clearly detected up to $40 \mathrm{keV}$, so spectral bins at higher energies are ignored. A power law fit to the ISGRI spectrum returns a relatively steep photon index of $4.5 \pm 0.4\left(\chi_{v}^{2}=0.32\right)$.

Spectral extraction for EPIC/PN, EPIC/MOS1, and EPIC/MOS2 relied on single and double events within a circle of radius $25^{\prime \prime}$ centred on the source. To estimate the background, we made an event list from a circle of equal radius in the same detector and at an equivalent distance from the detector's edge. Channels were configured to collect 30 counts per bin. An absorbed power law fit to the EPIC spectra has a photon index of $1.0 \pm 0.1$ with a column density of $3.1 \pm 0.1 \times 10^{23} \mathrm{~cm}^{-2}\left(\chi_{v}^{2}=1.15\right)$.

The EPIC spectrum is heavily-absorbed below $4 \mathrm{keV}$. Iron emission lines appear at $6.4 \mathrm{keV}\left(\mathrm{Fe} \mathrm{K}_{\alpha}\right)$ and at $7.1 \mathrm{keV}$ $\left(\mathrm{Fe} \mathrm{K}_{\beta}\right)$. A discontinuity in the continuum above $7.1 \mathrm{keV}$ suggests an iron absorption edge. There is an indication of an excess of soft emission between 0.5 and $2 \mathrm{keV}$. Including a soft blackbody component in an absorbed power law adjusted to the EPIC spectra decreases the $\chi^{2}$ by 9 for 680 degrees of freedom (d.o.f.), or a $0.5 \%$ probability that this feature is due to chance. Using a partial covering absorption instead of a blackbody raises the $\chi^{2}$ by 60 for the same dof (679). A partiallyionised absorber is also insufficient to model the soft excess.

Simply fitting an absorbed power law to the spectra from EPIC and ISGRI yields $N_{\mathrm{H}} \sim 4 \times 10^{23} \mathrm{~cm}^{-2}$ and $\Gamma \sim 2.3$, but the fit is poor $\left(\chi_{v}^{2}=2.1\right)$ with significant residuals in the hard $\mathrm{X}$-rays. The EPIC photon index of $1.0 \pm 0.1$ is smaller than the one observed for ISGRI $(4.5 \pm 0.4)$ which indicates a spectral break. Such spectral shapes - a flat power law at low energies with a high-energy cutoff between $10-20 \mathrm{keV}$ beyond which the slope steepens - are typical of X-ray pulsars (White et al. 1995).

We fit the combined EPIC and ISGRI spectra (Fig. 8) with a broken power law (BP), a power law with an exponential cutoff energy (CP), and a Compton emission (CE) model (comptt within Xspec). The models are modified by a galactic absorption in the direction of the source (fixed at $2.2 \times 10^{22} \mathrm{~cm}^{-2}$, Dickey \& Lockman 1990), and an intrinsic photoelectric absorption with free iron abundances $\left(Z_{\mathrm{Fe}}\right)$. Soft excess emission is represented by a blackbody (with a fixed temperature of $k T_{\mathrm{b}}=0.06 \mathrm{keV}$ ) that is affected by the galactic absorption only. Two narrow Gaussians (with widths fixed at 0 ) describe the iron lines. A constant $\left(C_{I}\right)$ accounts for the asynchronous observations and for cross-calibration uncertainties.

The BP model $\left(\chi_{v}^{2} /\right.$ d.o.f. $\left.=0.95 / 675\right)$ has photon indices of $\Gamma_{1}=0.9_{-0.2}^{+0.1}$ and $\Gamma_{2}=4.6_{-0.3}^{+0.8}$. However, the BP model does not constrain the break energy $\left(E_{\text {break }}>17 \mathrm{keV}\right)$ nor $C_{I}$ because it requires one more parameter than the other models. The CP model $\left(\chi_{v}^{2} /\right.$ d.o.f. $\left.=1.07 / 676\right)$ gives $\Gamma=0.8 \pm 0.2$, 


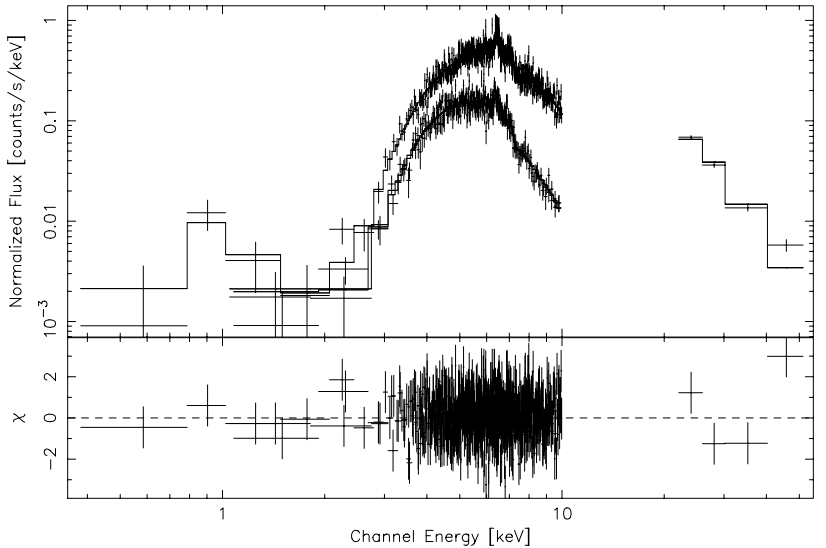

Fig. 8. Photon spectrum of IGR J16393-4643 from $E P I C / P N$, EPIC/MOS1, EPIC/MOS2, and ISGRI, fit with the comptonised continuum model (CE).

and a cutoff energy of $E_{\text {cut }}=10 \pm 1 \mathrm{keV}$, but residuals remain at high energy because of the low cutoff temperature. In Lutovinov et al. (2005a), an absorbed cutoff power law fit to the combined ASCA and ISGRI spectra had a comparable cutoff energy of $11 \pm 1 \mathrm{keV}$, but the photon index was poorly constrained $(\Gamma=1.3 \pm 1.0)$.

Parameters from the $\mathrm{CE}$ model $\left(\chi_{v}^{2} /\right.$ d.o.f. $\left.=0.95 / 676\right)$ are listed in Table 2 . The column density $\left(N_{\mathrm{H}}\right)$ is $(25 \pm 2) \times$ $10^{22} \mathrm{~cm}^{-2}$. The comptonising medium has an electron temperature $\left(k T_{\mathrm{e}}\right)$ of $4.4 \pm 0.3 \mathrm{keV}$ with an optical depth $(\tau)$ of $9 \pm 1$. The $\mathrm{Fe}_{\alpha}$ line is at $6.41 \pm 0.03$ and has an equivalent width of $60 \pm 30 \mathrm{eV}$ when measured with respect to the unabsorbed continuum, while the energy of the $\mathrm{Fe} \mathrm{K}_{\beta}$ line is $7.1 \pm 0.1 \mathrm{keV}$ with an equivalent width of $<120 \mathrm{eV}$. The detection of the $\mathrm{K}_{\beta}$ line is marginal given the uncertainties and its F-test probability of 3\%. However, the ratio of iron intensities $\left(F_{\mathrm{K} \beta} / F_{\mathrm{K} \alpha}\right)$ is consistent with the value expected from the photoionisation of iron (Kaastra \& Mewe 1993). The absorbed, integrated fluxes (in units of $10^{-11} \mathrm{erg} \mathrm{cm}^{-2} \mathrm{~s}^{-1}$ ) are 4.4 in the $2-10 \mathrm{keV}$ band, and 5.1 in the $20-60 \mathrm{keV}$ band. The observed soft excess flux between 0.5 and $2 \mathrm{keV}$ is $7 \times 10^{-15} \mathrm{erg} \mathrm{cm}^{-2} \mathrm{~s}^{-1}$. Since $C_{I} \sim 1$, the EPIC observation occurred during a period in which the source was in an average state.

\subsection{Phase-resolved spectrum}

The EPIC/PN spectra were binned according to two states: a pulse state around the primary peak in the folded light curve (phase $0.43-0.77$ in Fig. 7b), and a plateau state (phases $0.00-0.07$ and $0.87-1.00$ ). Average count rates for the pulse and plateau states are almost a factor of 2 apart at $\sim 1.2$ and $\sim 0.7$, respectively. The CE model was applied to the phaseresolved EPIC spectra constrained with the ISGRI spectrum. Bins for the plateau gather 20 counts and bins for the pulse collect 40 counts per channel. The soft excess is not prominent in either spectrum so the blackbody component is omitted. This prevents an evaluation of the influence of the pulse on the soft excess. Table 2 reports parameters for the CE model

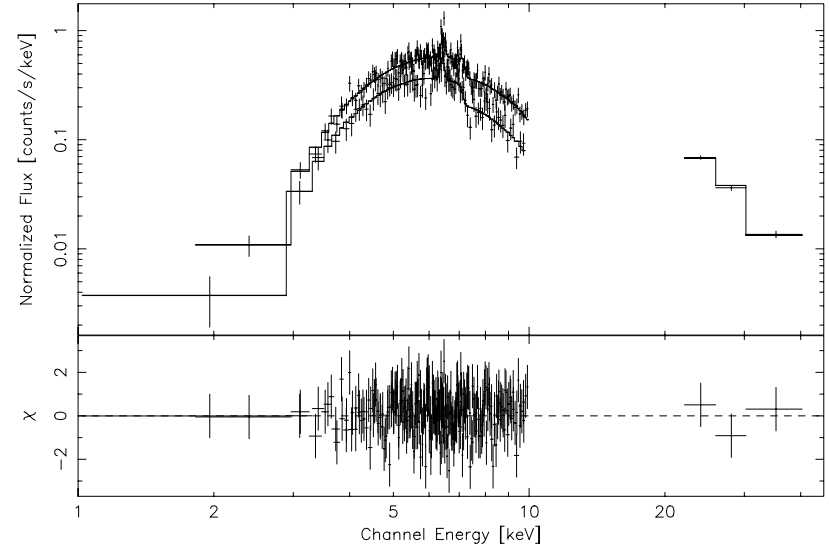

Fig. 9. Phase-resolved spectrum of IGR J16393-4643 from EPIC/PN during the pulse and plateau states. The ISGRI spectrum is included to constrain the $\mathrm{CE}$ model.

Table 2. Parameters from the Compton emission (CE) model fit to the spectra of EPIC and ISGRI combined. Also listed are the values of the CE model (without a blackbody) fit to the pulse and plateau states of the phase-resolved EPIC/PN spectra constrained with the ISGRI spectrum. The integrated fluxes are listed as observed $\left(F_{2-10}\right)$ or unabsorbed $\left(F_{2-10}^{\mathrm{UA}}\right)$. Errors denote $90 \%$ confidence.

\begin{tabular}{lcccl}
\hline \hline Parameter & CE & Pulse & Plateau & Unit \\
\hline$C_{I}$ & $0.8 \pm 0.2$ & $0.6 \pm 0.2$ & $1.3 \pm 0.7$ & \\
$\tau$ & $9 \pm 1$ & $12_{-2}^{+6}$ & $8_{-2}^{+4}$ & \\
$k T_{\mathrm{e}}$ & $4.4 \pm 0.3$ & $4.4 \pm 0.3$ & $4.4 \pm 0.4$ & $\mathrm{keV}$ \\
$N_{\mathrm{H}}$ & $25 \pm 2$ & $23_{-6}^{+2}$ & $24_{-7}^{+3}$ & $10^{22} \mathrm{~cm}^{-2}$ \\
$Z_{\mathrm{Fe}}$ & $1.0_{-0.2}^{+0.4}$ & $0.9_{-0.3}^{+0.6}$ & $1.2_{-0.6}^{+0.8}$ & $Z_{\odot}$ \\
$F_{2-10}$ & 4.4 & 5.2 & 3.0 & $10^{-11} \mathrm{erg} \mathrm{cm}^{-2} \mathrm{~s}^{-1}$ \\
$F_{2-10}^{\mathrm{UA}}$ & 9.2 & 9.8 & 6.7 & $10^{-11} \mathrm{erg} \mathrm{cm}^{-2} \mathrm{~s}^{-1}$ \\
$E_{\mathrm{K} \alpha}$ & $6.41 \pm 0.03$ & $6.44 \pm 0.03$ & $6.41 \pm 0.04$ & $\mathrm{keV}$ \\
$F_{\mathrm{K} \alpha}$ & $1.1 \pm 0.2$ & $1.1 \pm 0.4$ & $0.8 \pm 0.4$ & $10^{-4} \mathrm{ph} \mathrm{cm}^{-2} \mathrm{~s}^{-1}$ \\
$E_{\mathrm{K} \beta}$ & $7.1 \pm 0.1$ & $7.1 \pm 0.3$ & $7.2 \pm 0.3$ & $\mathrm{keV}$ \\
$F_{\mathrm{K} \beta}$ & $<0.62$ & $<1.1$ & $<1.3$ & $10^{-4} \mathrm{ph} \mathrm{cm}^{-2} \mathrm{~s}^{-1}$ \\
$\chi_{v}^{2} /$ d.o.f. & $0.95 / 676$ & $0.92 / 140$ & $0.92 / 94$ & \\
\hline
\end{tabular}

fit to the phase-resolved spectra. The pulsation affects the normalisations but does not modify the shape of the spectra nor its parameters, specifically the $N_{\mathrm{H}}$, in any appreciable way.

\section{Discussion}

Observations of IGR J16393-4643 by INTEGRAL and $X M M-N e w t o n$ present a source that is highly-obscured and persistent, with an intensity varying by a factor larger than 20 . The source has a pulsation period of $912.0 \pm 0.1 \mathrm{~s}$. These attributes, along with its spectral shape, its neutral iron lines, and its lack of radio emission, suggest a HMXB system consisting of a magnetised neutron star orbiting inside the dense stellar wind of a supergiant companion. In the $2-10 \mathrm{keV}$ energy band, the unabsorbed flux of IGR J16393-4643 is 
$9.2 \times 10^{-11} \mathrm{erg} \mathrm{cm}^{-2} \mathrm{~s}^{-1}$. Assuming a luminosity of $1.2 \times$ $10^{36} \mathrm{erg} \mathrm{s}^{-1}$, which is typical for accretion-driven X-ray pulsars (Bildsten et al. 1997), the source is located approximately $10 \mathrm{kpc}$ away.

The absorbing column density is large whenever the source is detected, whether by ASCA $\left(N_{\mathrm{H}}=13_{-7}^{+9} \times 10^{22} \mathrm{~cm}^{-2}\right)$, or now with INTEGRAL and XMM-Newton $\left(N_{\mathrm{H}}=(25 \pm 2) \times\right.$ $10^{22} \mathrm{~cm}^{-2}$ ). The strong absorption below $5 \mathrm{keV}$ is intrinsic since it is an order of magnitude larger than the galactic absorption along the line of sight $\left(N_{\mathrm{H}}=2.2 \times 10^{22} \mathrm{~cm}^{-2}\right)$. The absorption does not vary with the pulsation, which means that it is not related to the accretion column, but it may change with the orbital phase. This absorption indicates the presence of optically-thick material surrounding the compact object, which is consistent with the detection of iron lines.

Emission lines at 6.4 and $7.1 \mathrm{keV}$ trace the quantity of matter in the shell that envelopes the neutron star. These lines are at the positions that would be expected from the fluorescence of cold neutral matter illuminated by continuum X-rays, and their equivalent widths are compatible with a spherical distribution of dense gas around the compact object (Matt 2002). A K $\alpha$ line at $6.41 \pm 0.03 \mathrm{keV}(\gtrsim 1.925 \AA)$ corresponds to an ionisation of at most Fe XVIII (House 1969), which does not constrain the distance between the ionising source and the inner surface of the ionised shell. Given the errors on the line equivalent widths, we can not determine whether the fluxes of the iron lines are modified by the pulse.

There appears to be a soft excess flux of $7 \times$ $10^{-15} \mathrm{erg} \mathrm{cm}^{-2} \mathrm{~s}^{-1}$ that is best represented by a blackbody rather than with a partial covering or partially-ionised absorber. The scattering of X-rays by the stellar wind is the most likely explanation for the soft excess emission (Haberl \& White 1990). To what extent the pulse affects the soft excess emission is still unknown as the soft excess flux is not prominent in the phase-resolved spectrum.

The ASCA Faint Source Catalog (Sugizaki et al. 2001) listed IGR J16393-4643 among the brightest objects in its catalog, but the uncertainty of the position and the heavy absorption prevented Sugizaki et al. (2001) from associating this source with an optical counterpart. Malizia et al. (2004) noted the possible association between IGR J16393-4643 and the unidentified EGRET source 3EGJ1639-4702 (Hartman et al. 1999). Non-thermal radio emission was recently detected within the ASCA error box (Combi et al. 2004) which suggests a dust-enshrouded microquasar and could help justify the IBIS and EGRET association. However, the XMM-Newton position $\left(\mathrm{RA}(\mathrm{J} 2000)=16^{\mathrm{h}} 39^{\mathrm{m}} 05.4^{\mathrm{s}}\right.$, Dec $=-46^{\circ} 42^{\prime} 12^{\prime \prime}$, $4^{\prime \prime}$ uncertainty) is incompatible with the position of the radio source MOST J1639.0-4642, or any known radio source from the Vizier database. The pulsation and the lack of radio emission invalidate the microquasar interpretation for the HMXB. Also, the chance association of the EGRET source with IGRJ16393-4643 is strong given the high density of sources in the region.

A single infrared candidate, 2MASS J16390535-4642137, lies within the XMM-Newton error circle of IGR J16393-4643. The high-energy spectral and timing characteristics of IGR J16393-4643 are reminiscent of other heavily-absorbed, wind-accreting X-ray pulsars with OB supergiant companions (Walter et al. 2004). A Be stellar companion is unlikely given that such systems are usually transient. Infrared observations should confirm the supergiant nature of the companion. If this is the case, it will constitute another argument rejecting the EGRET association (Orellana \& Romero 2005).

Recently, combined INTEGRAL and XMM-Newton observations of the Norma Arm have revealed more pulsating X-ray binaries than were previously known. These objects have joined the ranks of what might be a new class of heavily-absorbed HMXBs that were previously undetected below $5 \mathrm{keV}$. The increasing sample size these objects represent should enable meaningful statistical studies to be performed. Understanding the nature of sources such as IGR J16393-4643 could shed light on the structure of stellar winds, provide constraints on the masses of neutron stars, and help elucidate the evolution of binaries.

Acknowledgements. The authors thank the anonymous referee for their prompt report which improved the paper. A. Bodaghee acknowledges J. Wendt, J. Rodriguez, A. Paizis, D. Willis, N. Produit and S. Paltani for their input and discussions. This research has made use of the SIMBAD database, operated at CDS, Strasbourg, France. This publication uses observations obtained with the ESA science missions INTEGRAL and XMM-Newton. The INTEGRAL and XMM-Newton instruments and data centres were directly funded by ESA member states and the USA (NASA).

\section{References}

Bildsten, L., Chakrabarty, D., Chiu, J., et al. 1997, ApJS, 113, 367

Bird, A. J., Barlow, E. J., Bassani, L., et al. 2004, ApJ, 607, L33

Combi, J. A., Ribó, M., Mirabel, I. F., \& Sugizaki, M. 2004, A\&A, 422, 1031

Courvoisier, T. J.-L., Walter, R., Beckmann, V., et al. 2003, A\&A, 411, L53

Cutri, R. M., Skrutskie, M. F., van Dyk, S., et al. 2003, VizieR Online Data Catalog, 2246, 0

Dickey, J. M., \& Lockman, F. J. 1990, ARA\&A, 28, 215

Ebisawa, K., Bourban, G., Bodaghee, A., Mowlavi, N., \& Courvoisier, T. J.-L. 2003, A\&A, 411, L59

Haberl, F., \& White, N. E. 1990, ApJ, 361, 225

Hartman, R. C., Bertsch, D. L., Bloom, S. D., et al. 1999, ApJS, 123, 79

Hill, A. B., Walter, R., Knigge, C., et al. 2005, A\&A, 411, L53

Horne, J. H., \& Baliunas, S. L. 1986, ApJ, 302, 757

House, L. L. 1969, ApJS, 18, 21

Jansen, F., Lumb, D., Altieri, B., et al. 2001, A\&A, 365, L1

Kaastra, J. S., \& Mewe, R. 1993, A\&AS, 97, 443

Lebrun, F., Leray, J. P., Lavocat, P., et al. 2003, A\&A, 411, L141

Liu, Q. Z., van Paradijs, J., \& van den Heuvel, E. P. J. 2000, A\&AS, 147,25

Lutovinov, A., Revnivtsev, M., Gilfanov, et al. 2005a, A\&A, 444, 821

Lutovinov, A., Rodriguez, J., Revnivtsev, M., \& Shtykovskiy, P. 2005b, A\&A, 433, L41

Malizia, A., Bassani, L., di Cocco, G., et al. 2004, The Astronomer's Telegram, 227, 1

Matt, G. 2002, MNRAS, 337, 147

Monet, D. G., Levine, S. E., Canzian, B., et al. 2003, AJ, 125, 984 
Ochsenbein, F., Bauer, P., \& Marcout, J. 2000, A\&AS, 143, 23

Orellana, M., \& Romero, G. E. 2005, Ap\&SS, 297, 167

Rodriguez, J., Tomsick, J. A., Foschini, L., et al. 2003, A\&A, 407, L41

Strüder, L., Briel, U., Dennerl, K., et al. 2001, A\&A, 365, L18 Sugizaki, M., Mitsuda, K., Kaneda, H., et al. 2001, ApJS, 134, 77 Turner, M. J. L., Abbey, A., Arnaud, M., et al. 2001, A\&A, 365, L27 Ubertini, P., Lebrun, F., Di Cocco, G., et al. 2003, A\&A, 411, L131 van Paradijs, J. 1983, in Accretion-Driven Stellar X-ray Sources, 189 Voges, W., Aschenbach, B., Boller, T., et al. 1999, A\&A, 349, 389 Walter, R., Rodriguez, J., Foschini, L., et al. 2003, A\&A, 411, L427
Walter, R., Courvoisier, T. J.-L., Foschini, L., et al. 2004, in The INTEGRAL Universe, Proceedings of the Fifth INTEGRAL Workshop, 16-20 February 2004, Munich, Germany, ed. B. Battrick, Scientific ed. V. Schoenfelder, G. Lichti, \& C. Winkler, ESA SP-552 (Noordwijk: ESA Publication Division), 417

White, N. E., Nagase, F., \& Parmar, A. N. 1995, X-ray binaries Cambridge Astrophysics Series, ed. W. H. G. Lewin, J. Van Paradijs, \& E. P. J. Van den Heuvel (Cambridge, MA: Cambridge University Press), 1

Winkler, C., Courvoisier, T. J.-L., Di Cocco, G., et al. 2003, A\&A, 411, L1 